\title{
Smartphone Addiction and Associated Health Outcomes in Adult Populations: A Systematic Review
}

\author{
Zubair Ahmed Ratan ${ }^{1,2}$, Anne-Maree Parrish ${ }^{1, *(\mathbb{D})}$, Sojib Bin Zaman ${ }^{3} \mathbb{D}$, Mohammad Saud Alotaibi ${ }^{1,4}(\mathbb{D}$ and \\ Hassan Hosseinzadeh $1, *$ (D)
}

1 School of Health and Society, Faculty of the Arts, Social Sciences and Humanities, University of Wollongong, Northfields Ave., Wollongong, NSW 2522, Australia; zar241@uowmail.edu.au (Z.A.R.); ma098@uowmail.edu.au (M.S.A.)

2 Department of Biomedical Engineering, Khulna University of Engineering and Technology, Khulna 9203, Bangladesh

3 Department of Medicine, School of Clinical Sciences at Monash Health, Monash University, Melbourne, VIC 3800, Australia; sojib.zaman@monash.edu

4 Department of Social Work, College of Social Sciences, Umm Al-Qura University, Mecca 24382, Saudi Arabia

* Correspondence: aparrish@uow.edu.au (A.-M.P.); hassanh@uow.edu.au (H.H.)

\section{check for} updates

Citation: Ratan, Z.A.; Parrish, A.-M.; Zaman, S.B.; Alotaibi, M.S.; Hosseinzadeh, H. Smartphone Addiction and Associated Health Outcomes in Adult Populations: A Systematic Review. Int. J. Environ. Res. Public Health 2021, 18, 12257. https://doi.org/10.3390/ ijerph182212257

Academic Editors: Elisardo Becoña Iglesias and Paul B. Tchounwou

Received: 1 October 2021

Accepted: 18 November 2021

Published: 22 November 2021

Publisher's Note: MDPI stays neutral with regard to jurisdictional claims in published maps and institutional affiliations.

Copyright: (c) 2021 by the authors. Licensee MDPI, Basel, Switzerland. This article is an open access article distributed under the terms and conditions of the Creative Commons Attribution (CC BY) license (https:// creativecommons.org/licenses/by/ $4.0 /)$

\begin{abstract}
Background: Smartphones play a critical role in increasing human-machine interactions, with many advantages. However, the growing popularity of smartphone use has led to smartphone overuse and addiction. This review aims to systematically investigate the impact of smartphone addiction on health outcomes. Methods: The Preferred Reporting Items for Systematic Reviews and Meta-Analyses (PRISMA) guidelines were used to carry out the systematic review. Five electronic databases including Medline, Web of Science, PsycINFO, PubMed, and Scopus were searched to identify eligible studies. Eligible studies were screened against predetermined inclusion criteria and data were extracted according to the review questions. This review is registered in PROSPERO (CRD42020181404). The quality of the articles was assessed using the National Institutes of Health (NIH) Quality Assessment Tool for Observational Cohort and Cross-Sectional Studies. Results: A total of 27 of 2550 articles met the inclusion criteria. All of the studies were cross-sectional and focused on physical, mental, and neurological health outcomes. The majority of the studies focused on mental health outcomes and consistent associations were observed between smartphone addiction and several mental health outcomes. Anxiety and depression were commonly found to mediate mental health problems. A wide range of physical health sequelae was also associated with smartphone addiction. Furthermore, there was an association between smartphone addiction and neurological disorders. Conclusions: Our findings suggest that there are consistent associations between smartphone addiction and physical and mental health, especially mental health. Social awareness campaigns about smartphone addiction and its impact on physical and mental health are needed. Further studies, especially randomized controlled trials, are warranted to validate the impacts of smartphone addiction.
\end{abstract}

Keywords: smartphone; addiction; health outcomes

\section{Introduction}

The 21st century is known as the age of information technology. Wireless communication and the internet are remarkable entities resulting in revolutionary changes in the field of communication [1]. In 2007, computer-based phones (smartphones) were introduced [2]. Since then, smartphones have become an indispensable part of daily life in all communities and countries. As such, smartphones have become one of the fastest-growing sectors in the technology industry [3]. Over the past decade, smartphone ownership and use have been exponentially increased globally. For instance, there were about 2.1 billion smartphone users in 2017 and the number was projected to exceed 2.8 billion by 2020 worldwide [4]. 
A number of novel problematic behaviors have emerged in the information technology era, such as gambling, internet gaming, and sexual behaviors, which may lead to compulsive engagement [5]. Extreme instances may lead to individuals feeling unable to control these behaviors without external influence, and these behaviors may be considered non-substance or behavioral addictions [6]. Internet addiction is one of the earliest examined forms of information technology addiction [7]. The relatively newer concept of "smartphone addiction" (SA) has also been studied based on previous internet addiction research [8]. Smartphones distinguish their use from traditional Internet use on computers or laptops because smartphones allow users to access the internet continuously regardless of time and space. Smartphone addiction is fueled by an Internet overuse problem or Internet addiction disorder [9]. The increased use of smartphones has resulted in most in people communicating daily online, as a result of interactive texts and social media, instead of face-to-face human contact. Smartphones fetch a limitless range of cognitive activities for users; smartphones forge opportunities for individuals to engage in a range of online activities such as participating in social network sites, playing video games, and "surfing the web" [10]. However, the smartphone poses a negative impact on our ability to think, remember, pay attention, and regulate emotion [11]. The increase in popularity and frequency of smartphone use has led to the emergence of clinical cases of people presenting with abuse symptoms [12].

The concept of addiction is not easy to define, and the usage of the term addiction has been considered controversial; however, central to its definition is the dependence on a substance or activity [13].

Smartphone addiction (SA) is generally conceptualized as a behavioral addiction including mood tolerance, salience, withdrawal, modification, conflict, and relapse [14]. Literature suggests that there are associations between SA and mental health [15], physical health [16], and neurological problems [17]. Furthermore, tolerance, salience, withdrawal, and cravings $[8,18]$ have been associated with excessive smartphone use. However, the evidence is not conclusive [19]. Still, there is debate in the literature about the positive or negative relationship between the amount of screen time or smartphone use and health outcomes. Existing studies have provided useful data; however, it is difficult to draw consensus without a systematic review.

This systematic review is an attempt to collate empirical evidence about the health impacts of smartphone addiction among the adult population. This study aims to provide evidence to inform policy or recommendations to control and prevent smartphone addiction.

\section{Methods}

The protocol of this systematic review is registered in PROSPERO (CRD42020181404). It was carried out using the PRISMA (Preferred Reporting Items for Systematic Reviews and Meta-Analyses) guidelines (Figure 1). Literature searches were conducted in the five databases including Scopus, Medline, PubMed, Web of Science, and psycINFO databases. The search strategy for this review was initially developed by a series of consultations with the investigators and some preliminary searches (Z.A.R., A.M.P., S.B.Z., M.S.A., and H.H.). Expert librarians from the University of Wollongong were also consulted to refine and finalize the search strategy. All studies including controlled trials, case-control, crosssectional, and cohort studies were included. Eligibility criteria included studies which explored smartphone exposure focusing on the adult population (aged over 18), published in the English language. This review excluded case reports, ideas, editorials, meta-analysis, review articles and opinions. Search terms included "smartphone", "addiction", "overuse", "problematic use", "excessive use", and "adults". Details of search strategies are provided in Supplementary Table S1. Since the smartphone gained popularity in 2011 (after the debut of the smartphone), the literature was searched from January 2011 until July 2021. The reference lists of the selected papers were also searched for any eligible papers however no papers were found. 

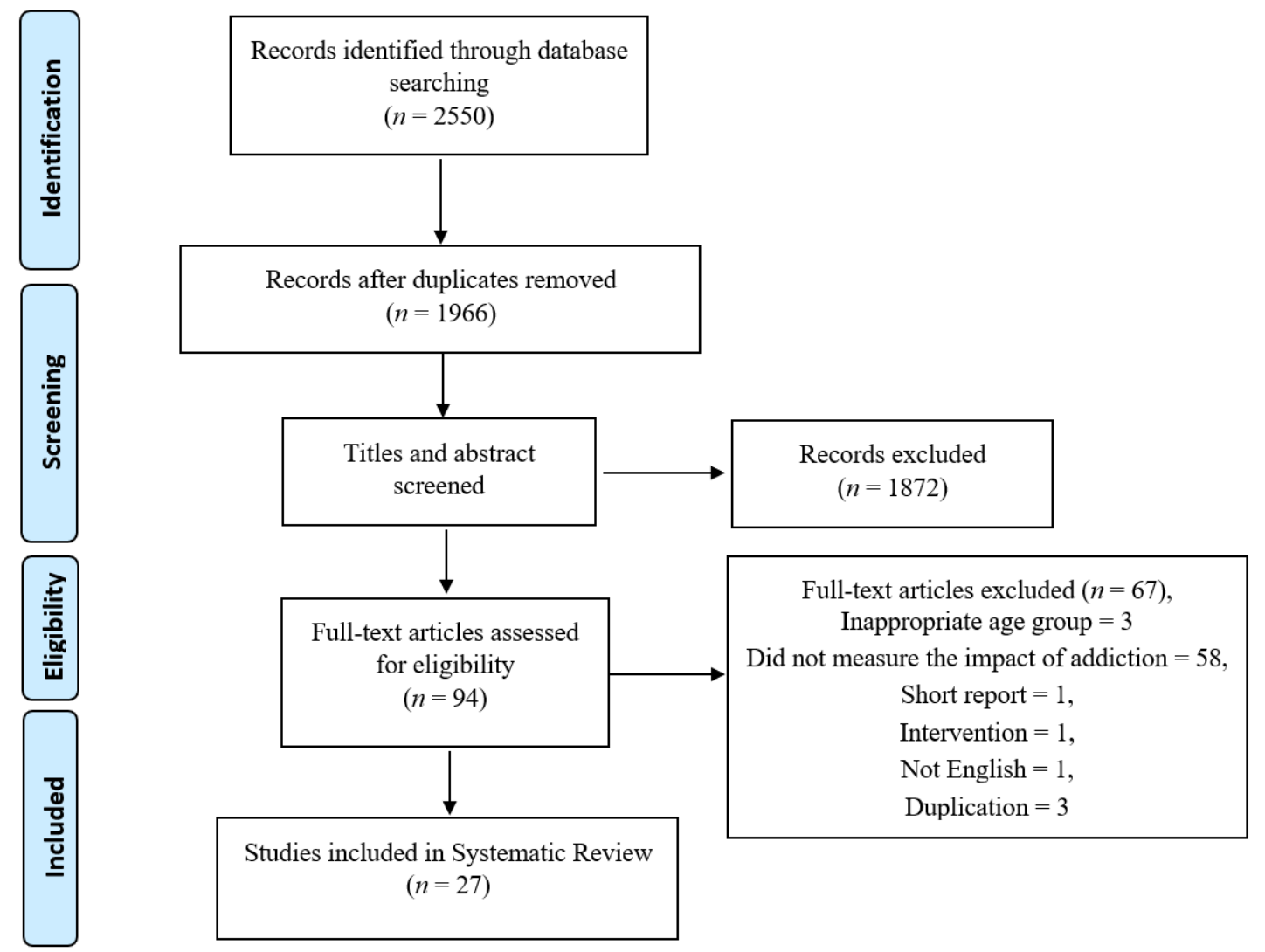

Figure 1. Preferred Reporting Item for Systematic Review (template taken from PRISMA flow diagram).

Three authors (Z.A.R., S.B.Z., and M.S.A.) independently reviewed all the retrieved abstracts and selected eligible papers. Any disagreements were resolved by discussion with senior researchers (A.M.P. and H.H.). The quality of each included study was assessed by using the National Institutes of Health (NIH) Quality Assessment Tool for Observational Cohort and Cross-Sectional Studies and were given a rating of either "good", "fair" or "poor" and the results of the quality assessment are presented in Supplementary Table S2. The NIH quality assessment is a valid and reliable tool for the assessment of the methodological quality of cross-sectional studies [20].

\section{Results}

\subsection{Overall Search Findings}

A total of 2550 potential studies were identified. After screening and removing duplicates, twenty-seven (27) studies were eligible for this review. A detailed study selection process based on the PRISMA flow chart is presented in Figure 1. Sample sizes ranged from 30 to 5372 adults (Table 1). Seven were conducted in South Korea [21-27], three in Saudi Arabia [28-30], four in China [31-34], four in Turkey [35-38], one in India [39] one in Taiwan [40], one in Switzerland [41], one in the USA [42], one in Italy [43], one in Thailand [44], and three were international studies [45-47] (Figure 2). Smartphone addiction was measured in the study sample using different scales, however, the Smartphone Addiction Scale, Short Version (SAS-SV; $n=8$ ) was the most common measure (Table 1). Among the selected studies, nine studies were considered to be "good", seventeen articles were considered to be "fair", and the remaining one was considered "poor" (Table 2). 
Table 1. Smartphone addiction and associated health outcomes.

\begin{tabular}{|c|c|c|c|c|c|c|c|c|}
\hline $\begin{array}{c}\text { Authors, Country, } \\
\text { Year }\end{array}$ & $\begin{array}{l}\text { Sample } \\
\text { Size }\end{array}$ & $\begin{array}{c}\text { Type of } \\
\text { Population }\end{array}$ & Age/Age Range & Gender & Type of Study & Outcome Measurement Tool & Pattern of Survey & $\begin{array}{l}\text { Assessment } \\
\text { Tool (SA) }\end{array}$ \\
\hline $\begin{array}{c}\text { Hye-Jin } \\
\text { Kim [21,47], } \\
\text { South Korea, } \\
2017\end{array}$ & 608 & $\begin{array}{l}\text { University/college } \\
\text { students }\end{array}$ & $\begin{array}{l}\text { Control:23.01 } \pm 2.32 \\
\text { SA: } 22.54 \pm 2.05\end{array}$ & $\begin{array}{c}\text { Male }=183 \\
\text { Female }=425\end{array}$ & Cross-sectional & $\begin{array}{l}\text { Self-reported experience of } \\
\text { accidents was assessed }\end{array}$ & $\begin{array}{c}\text { Online } \\
\text { questionnaire-based } \\
\text { survey }\end{array}$ & SAPS \\
\hline $\begin{array}{l}\text { Yeon-Jin Kim [22], } \\
\text { South Korea, } \\
2015\end{array}$ & 4854 & General & Age range 19-49 & $\begin{array}{c}\text { Male }=2573 \\
\text { Female }=2281\end{array}$ & Cross-sectional & $\begin{array}{l}\text { The Symptom Checklist-90- } \\
\text { Revised-SCL-90-R }\end{array}$ & Online survey & K-scale \\
\hline $\begin{array}{l}\text { Deokjong Lee [23], } \\
\text { South Korea, } \\
2019\end{array}$ & 94 & General & $\begin{array}{c}22.6 \pm 2.4 \\
\text { (Age range 16-27) }\end{array}$ & $\begin{array}{c}\text { Male }=61 \\
\text { Female }=27\end{array}$ & Cross-sectional & $\begin{array}{l}\text { Magnetic resonance imaging } \\
\text { (MRI) scan }\end{array}$ & $\begin{array}{c}\text { Online } \\
\text { advertisements, } \\
\text { MRI }\end{array}$ & SAPS \\
\hline $\begin{array}{l}\text { JeonHyeong } \\
\text { Lee [24], } \\
\text { South Korea, } \\
2014\end{array}$ & 30 & $\begin{array}{l}\text { University } \\
\text { students }\end{array}$ & $\begin{array}{c}\mathrm{N}=22.6 \pm 1.3 \\
\text { Moderate } \\
\text { Addiction Group } \\
(\mathrm{MAG})=21.5 \pm 1.9 \\
\text { Severe Addiction } \\
\text { Group } \\
(\mathrm{SAG})=22.4 \pm 2.0\end{array}$ & $\begin{array}{l}\text { Male }=12 \\
\text { Female }=18\end{array}$ & Cross-sectional & $\begin{array}{c}\text { Motion meter (Performance } \\
\text { Attainment Associates, West } \\
\text { Germany) }\end{array}$ & $\begin{array}{l}\text { Survey, the range of } \\
\text { motion (ROM), a } \\
\text { range of motion } \\
\text { meter (Performance } \\
\text { Attainment } \\
\text { Associates, West } \\
\text { Germany) }\end{array}$ & SAPS \\
\hline $\begin{array}{l}\text { Kyung Eun } \\
\text { Lee [25], } \\
\text { South Korea, } \\
2016\end{array}$ & 1261 & $\begin{array}{l}\text { University/ } \\
\text { college students }\end{array}$ & $\begin{array}{c}\text { M } 23.6 \pm 2.7 \\
\text { F } 21.5 \pm 2.7\end{array}$ & $\begin{array}{l}\text { Male }=725 \\
\text { Femle }=511\end{array}$ & $\begin{array}{l}\text { Cross-sectional } \\
\text { study }\end{array}$ & $\begin{array}{l}\text { Zung's Self-Rating Anxiety } \\
\text { Scale }\end{array}$ & $\begin{array}{l}\text { Face-to-face } \\
\text { interview }\end{array}$ & $\begin{array}{l}\text { Young's Internet } \\
\text { Addiction Test }\end{array}$ \\
\hline $\begin{array}{l}\text { Yeon-Seop } \\
\text { Lee [26], } \\
\text { South Korea, } \\
2012\end{array}$ & 125 & General & $21.4 \pm 2.0$ & $\begin{array}{c}\text { Male }=32 \\
\text { Female }=93\end{array}$ & Cross-sectional & $\begin{array}{c}\text { Phalen's tests, Reverse } \\
\text { Phalen's tests, } \\
\text { Ultrasonography }\end{array}$ & $\begin{array}{c}\text { Structured } \\
\text { questionnaires }\end{array}$ & $\begin{array}{c}\text { Structured } \\
\text { questionnaires }\end{array}$ \\
\hline $\begin{array}{c}\text { Mi Jung Rho [27] } \\
\text { South Korea, } \\
2019\end{array}$ & 5372 & General & $\begin{array}{c}26.43 \pm 5.954 \\
\text { (Age range 19-39) }\end{array}$ & $\begin{array}{c}\text { Male }=2443 \\
\text { Female }=2929\end{array}$ & Cross-sectional & $\begin{array}{c}\text { Brief Self-Control Scale } \\
\text { (BSCS), Generalized Anxiety } \\
\text { Disorder (GAD)-7, Patient } \\
\text { Health Questionnaire-9 } \\
\text { (PHQ-9), and Dickman } \\
\text { Impulsivity Inventory-Short } \\
\text { Version (DII). }\end{array}$ & Web survey & S-Scale \\
\hline
\end{tabular}


Table 1. Cont.

\begin{tabular}{|c|c|c|c|c|c|c|c|c|}
\hline $\begin{array}{c}\text { Authors, Country, } \\
\text { Year }\end{array}$ & $\begin{array}{l}\text { Sample } \\
\text { Size }\end{array}$ & $\begin{array}{c}\text { Type of } \\
\text { Population }\end{array}$ & Age/Age Range & Gender & Type of Study & Outcome Measurement Tool & Pattern of Survey & $\begin{array}{l}\text { Assessment } \\
\text { Tool (SA) }\end{array}$ \\
\hline $\begin{array}{c}\text { Aljohara A. } \\
\text { Alhassan [28], } \\
\text { Saudi Arabia, 2018 }\end{array}$ & 935 & General public & $\begin{array}{c}31.7 \pm 10.98 \\
\text { younger age group } \\
\text { (18-35 years), } \\
\text { middle-age group } \\
\text { (36-54 years), and } \\
\text { older age group } \\
(\geq 55 \text { years })\end{array}$ & $\begin{array}{c}\text { Male }=316 \\
(33.8 \%) \\
\text { Female }=619 \\
(66.2 \%)\end{array}$ & Cross-sectional & $\begin{array}{l}\text { The Beck's Depression } \\
\text { Inventory second edition }\end{array}$ & Web-based & SAS-SV \\
\hline $\begin{array}{l}\text { Alosaimi, F. } \\
\text { D. [29], } \\
\text { Saudi Arabia, } \\
2016\end{array}$ & 2367 & $\begin{array}{l}\text { University } \\
\text { students }\end{array}$ & not mentioned & Male $=43.6 \%$ & Cross-sectional & Not mentioned & $\begin{array}{l}\text { An electronic } \\
\text { self-administered } \\
\text { questionnaire }\end{array}$ & PUMP \\
\hline $\begin{array}{c}\text { Dalia } \\
\text { El-Sayed [30], } \\
\text { Saudi Arabia, 2020 }\end{array}$ & 1513 & $\begin{array}{l}\text { University } \\
\text { students }\end{array}$ & $\mathrm{M}=20.58(1.71)$ & $\begin{array}{c}\text { Male }=825(54.5 \%) \\
\text { Female }=688 \\
(45.5 \%)\end{array}$ & Cross-sectional & $\begin{array}{c}\text { Taylor Manifest Anxiety Scale } \\
\text { and Beck Depression } \\
\text { Inventory }\end{array}$ & Not reported & $\begin{array}{c}\text { The Problematic } \\
\text { Use of Mobile } \\
\text { Phones (PUMP) } \\
\text { scale }\end{array}$ \\
\hline $\begin{array}{l}\text { Jon D. Elhai [31], } \\
\text { China, } \\
2019\end{array}$ & 1034 & Young adults & $19.34 \pm 1.61$ & $\begin{array}{c}\text { Male }=359 \\
\text { Female }=675\end{array}$ & Cross-sectional & $\begin{array}{l}\text { Depression anxiety stress } \\
\text { scale-21 (DASS-21), Fear of } \\
\text { missing out (FOMO) scale }\end{array}$ & Web survey & SAS-SV \\
\hline $\begin{array}{l}\text { Yuanming } \mathrm{Hu}[32], \\
\text { China, } \\
2017\end{array}$ & 49 & Young adults & $\begin{array}{c}\text { Control: } \\
23.07 \pm 2.01, \text { SPD: } \\
22.11 \pm 1.78\end{array}$ & $\begin{array}{c}\text { Male }=26 \\
\text { Female }=23\end{array}$ & Cross-sectional & $\begin{array}{l}\text { Tract-based spatial statistics } \\
\text { (TBSS) analysis }\end{array}$ & $\begin{array}{c}\text { Survey } \\
\text { questionnaire }\end{array}$ & MPATS \\
\hline $\begin{array}{l}\text { Jon D. Elhai [33], } \\
\text { China, } \\
2020\end{array}$ & 908 & General & $\begin{array}{l}\text { Age averaged } \\
40.37 \text { years } \\
(\mathrm{SD}=9.27)\end{array}$ & $\begin{array}{c}\text { Male }=156 \\
\text { Female }=752\end{array}$ & Cross-sectional & $\begin{array}{c}\text { Depression anxiety stress } \\
\text { scale-21 (DASS-21) } \\
\text { Generalized anxiety disorder } \\
\text { scale-7 (GAD-7) for } \\
\text { COVID-19 anxiety }\end{array}$ & Web-based survey & $\begin{array}{l}\text { Smartphone } \\
\text { addiction } \\
\text { scale-short } \\
\text { version } \\
\text { (SAS-SV) }\end{array}$ \\
\hline $\begin{array}{l}\text { Linbo Zhuang [34], } \\
\text { China, } 2021\end{array}$ & 2438 & Young patients & Age, $18-44$ years & $\begin{array}{c}\text { Male }=1085 \\
\text { Female }=1353\end{array}$ & $\begin{array}{l}\text { Cross-sectional } \\
\text { study }\end{array}$ & $\begin{array}{c}\text { Magnetic Resonance Imaging } \\
\text { (MRI) examination, } \\
\text { Cervical Disc Degeneration } \\
\text { Scale (CDDS) }\end{array}$ & Not reported & $\begin{array}{l}\text { Smartphone } \\
\text { Addiction Scale } \\
\text { (SAS) }\end{array}$ \\
\hline
\end{tabular}


Table 1. Cont.

\begin{tabular}{|c|c|c|c|c|c|c|c|c|}
\hline $\begin{array}{c}\text { Authors, Country, } \\
\text { Year }\end{array}$ & $\begin{array}{l}\text { Sample } \\
\text { Size }\end{array}$ & $\begin{array}{c}\text { Type of } \\
\text { Population }\end{array}$ & Age/Age Range & Gender & Type of Study & Outcome Measurement Tool & Pattern of Survey & $\begin{array}{c}\text { Assessment } \\
\text { Tool (SA) }\end{array}$ \\
\hline $\begin{array}{l}\text { Yasemin P. } \\
\text { Demir [35], } \\
\text { Turkey, } \\
2019\end{array}$ & 123 & $\begin{array}{l}\text { Patients who had } \\
\text { Migraine }\end{array}$ & $\begin{array}{l}>18 \text { years and } \\
<65 \text { years }\end{array}$ & $\begin{array}{c}\text { Male }=69 \\
\text { Female }=54\end{array}$ & $\begin{array}{l}\text { Cross-sectional } \\
\text { comparative }\end{array}$ & $\begin{array}{c}\text { Migraine disability } \\
\text { assessment (MIDAS) } \\
\text { questionnaire, The Visual } \\
\text { Analogue Scale (VAS), } \\
\text { Migraine Quality of Life } \\
\text { Questionnaire) 24-h MQoLQ, } \\
\text { Pittsburgh Sleep Quality } \\
\text { Index (PSQI), Epworth } \\
\text { Sleepiness Scale (ESS) }\end{array}$ & $\begin{array}{l}\text { Written survey } \\
\text { questionnaire }\end{array}$ & PUMP \\
\hline $\begin{array}{l}\text { Kadir Demirci [36], } \\
\text { Turkey, } \\
2015\end{array}$ & 319 & $\begin{array}{l}\text { University } \\
\text { students }\end{array}$ & $\begin{array}{l}\text { Mean age }=20.5 \pm \\
2.45 \text { years } \\
\text { - Smartphone } \\
\text { non-user group } \\
20.8 \pm 2.11 \\
\text { - Low smartphone } \\
\text { use group } 20.7 \pm \\
2.74 \\
\text { - High smartphone } \\
\text { use group } 20.2 \pm \\
2.31\end{array}$ & $\begin{array}{l}\text { Male }=116 \\
\text { Female }=203\end{array}$ & Cross-sectional & $\begin{array}{c}\text { Pittsburgh Sleep Quality } \\
\text { Index (PSQI), Beck } \\
\text { Depression Inventory (BDI), } \\
\text { Beck Anxiety Inventory (BAI) }\end{array}$ & Not reported & PUMP \\
\hline $\begin{array}{l}\text { Ayse Gokce [37], } \\
\text { Turkey, } \\
2021\end{array}$ & 319 & $\begin{array}{l}\text { University } \\
\text { Students }\end{array}$ & $18-33,21.03 \pm 2.05$ & $\begin{array}{c}\text { Male }=104 \\
\text { Female }=215\end{array}$ & $\begin{array}{l}\text { Cross-sectional } \\
\text { study }\end{array}$ & $\begin{array}{l}\text { The Liebowitz Social Anxiety } \\
\text { Scale (LSAS); } \\
\text { Eating Attitudes Test (EAT). }\end{array}$ & Face-to-face survey & $\begin{array}{l}\text { Problematic } \\
\text { Mobile Phone } \\
\text { Use Scale }\end{array}$ \\
\hline $\begin{array}{l}\text { Betul Ozcan [38], } \\
\text { Turkey, } \\
2021\end{array}$ & 1545 & & $21.39 \pm 2.21$ years & $\begin{array}{c}\text { Male }=43.2 \% \\
\text { Female }=56.8 \%\end{array}$ & $\begin{array}{l}\text { Cross-sectional } \\
\text { study }\end{array}$ & $\begin{array}{l}\text { Pittsburgh Sleep Quality } \\
\text { Index (PSQI) }\end{array}$ & Not reported & $\begin{array}{c}\text { Smartphone } \\
\text { Addiction } \\
\text { Scale-Short } \\
\text { Version } \\
\text { (SAS-SV) }\end{array}$ \\
\hline
\end{tabular}


Table 1. Cont.

\begin{tabular}{|c|c|c|c|c|c|c|c|c|}
\hline $\begin{array}{c}\text { Authors, Country, } \\
\text { Year }\end{array}$ & $\begin{array}{l}\text { Sample } \\
\text { Size }\end{array}$ & $\begin{array}{c}\text { Type of } \\
\text { Population }\end{array}$ & Age/Age Range & Gender & Type of Study & Outcome Measurement Tool & Pattern of Survey & $\begin{array}{c}\text { Assessment } \\
\text { Tool (SA) }\end{array}$ \\
\hline $\begin{array}{l}\text { S HariPriya [39], } \\
\text { India, } \\
2019\end{array}$ & 113 & College students & $\begin{array}{c}22.15 \pm 1.69 \\
\text { (Age range 19-25) }\end{array}$ & $\begin{array}{c}\text { Male }=63 \\
\text { Female }=50\end{array}$ & $\begin{array}{l}\text { Cross-sectional } \\
\text { study }\end{array}$ & $\begin{array}{c}\text { Pittsburgh Sleep Quality } \\
\text { Index (PSQI), International } \\
\text { Physical Activity } \\
\text { Questionnaire-Short Form } \\
\text { (IPAQSF) }\end{array}$ & $\begin{array}{l}\text { Written survey } \\
\text { questionnaire }\end{array}$ & $\begin{array}{l}\text { Self-reported } \\
\text { questionnaire }\end{array}$ \\
\hline $\begin{array}{c}\text { Hsien-Yuan } \\
\text { Lane [40], Taiwan, } \\
2021\end{array}$ & 422 & $\begin{array}{l}\text { University } \\
\text { students }\end{array}$ & $\begin{array}{c}20.22(\mathrm{SD}=2.34 \\
\text { years })\end{array}$ & $\begin{array}{c}\text { Male }=79 \\
\text { Female }=343\end{array}$ & $\begin{array}{l}\text { Cross-sectional } \\
\text { study }\end{array}$ & $\begin{array}{c}\text { Tri-Dimensional Personality } \\
\text { Questionnaire (TPQ), } \\
\text { Chinese Version of the } \\
\text { Pittsburgh Sleep Quality } \\
\text { Index (CPSQI), } \\
\text { Beck Depression Inventory } \\
\text { (BDI), } \\
\text { Beck Anxiety Inventory (BAI) }\end{array}$ & Online & $\begin{array}{l}\text { Chen's } \\
\text { Smartphone } \\
\text { Addiction } \\
\text { Inventory }\end{array}$ \\
\hline $\begin{array}{c}\text { Anna Maria [41] } \\
\text { Switzerland, } 2021\end{array}$ & 240 & Young adults & $\begin{array}{l}18-35 \text { years old, } \\
\text { Mean age }=23.33\end{array}$ & $\begin{array}{c}\text { Male }=120 \\
\text { Female }=120\end{array}$ & Cross-sectional & $\begin{array}{l}\text { 12-item Social Anxiety Scale, } \\
\text { a question on the daily } \\
\text { duration of smartphone use, } \\
\text { a single-item measure of } \\
\text { dispositional truth }\end{array}$ & Online & $\begin{array}{l}\text { Smartphone } \\
\text { Addiction Scale } \\
\text { Short Version }\end{array}$ \\
\hline $\begin{array}{l}\text { Jon D. Elhai [42], } \\
\text { USA, } \\
2018\end{array}$ & 300 & College students & $19.87 \pm 3.79$ & $\begin{array}{c}\text { Male }=24.3 \% \\
\text { Female }=75.7 \%\end{array}$ & Cross-sectional & $\begin{array}{c}\text { Penn State Worry } \\
\text { Questionnaire-Abbreviated } \\
\text { Version (PSWQ-A), } \\
\text { Dimensions of Anger } \\
\text { Reactions-5 (DAR-5) Scale }\end{array}$ & Web survey & SAS-SV \\
\hline $\begin{array}{c}\text { Matteo } \\
\text { Megna [43], } \\
\text { Italy, } \\
2018\end{array}$ & 52 & Psoriatic patients & $\begin{array}{c}26.9 \pm 7.8 \text { (age } \\
\text { range 18-35) }\end{array}$ & $\begin{array}{c}\text { Male }=24 \\
\text { Female }=28\end{array}$ & Cross-sectional & $\begin{array}{l}\text { Nail Psoriasis Severity Index } \\
\text { (NAPSI), Early psoriatic } \\
\text { arthritis screening } \\
\text { questionnaire (EARP), } \\
\text { ultrasound score }\end{array}$ & $\begin{array}{l}\text { Face-to-face } \\
\text { interview }\end{array}$ & SAS-SV \\
\hline
\end{tabular}


Table 1. Cont.

\begin{tabular}{|c|c|c|c|c|c|c|c|c|}
\hline $\begin{array}{c}\text { Authors, Country, } \\
\text { Year }\end{array}$ & $\begin{array}{l}\text { Sample } \\
\text { Size }\end{array}$ & $\begin{array}{c}\text { Type of } \\
\text { Population }\end{array}$ & Age/Age Range & Gender & Type of Study & Outcome Measurement Tool & Pattern of Survey & $\begin{array}{c}\text { Assessment } \\
\text { Tool (SA) }\end{array}$ \\
\hline $\begin{array}{l}\text { Arunrat } \\
\text { Tangmunkongvo- } \\
\text { rakulI [44], } \\
\text { Thailand, } \\
2019 \\
\end{array}$ & 800 & $\begin{array}{l}\text { University } \\
\text { students }\end{array}$ & $\begin{array}{c}\text { 18-24 } \\
\text { (Age range 18-24) }\end{array}$ & $\begin{array}{c}\text { Male }=395 \\
\text { Female }=405\end{array}$ & Cross-sectional & Flourishing Scale (FS) & Face-to-face & $\begin{array}{r}\text { Young's Interne } \\
\text { Addiction Test }\end{array}$ \\
\hline $\begin{array}{c}\text { Zaheer } \\
\text { Hussain [45], } \\
\text { Global (majority in } \\
\text { the UK, 86\%), } \\
2017\end{array}$ & 640 & General & $\begin{array}{c}24.89 \pm 8.54 \\
\text { (Age range 13-69) }\end{array}$ & $\begin{array}{c}\text { Male }=214 \\
\text { Female }=420\end{array}$ & Cross-sectional & $\begin{array}{c}\text { Spielberger State-Trait } \\
\text { Anxiety Inventory (STAI) } \\
\text { Short-Form }\end{array}$ & Online survey & $\begin{array}{c}\text { Independent } \\
\text { questionnaire } \\
\text { (Problematic } \\
\text { smartphone use } \\
\text { scale) }\end{array}$ \\
\hline $\begin{array}{c}\text { Miles } \\
\text { Richardson [46], } \\
2018, \\
\text { Global (majority } \\
\text { UK, 82.8\%) }\end{array}$ & 244 & General & $29.72 \pm 12.16$ & $\begin{array}{c}\text { Male }=90 \\
\text { Female }=149\end{array}$ & Cross-sectional & $\begin{array}{l}\text { Spielberger State-Trait } \\
\text { Anxiety Inventory (STAI), } \\
\text { Nature Relatedness Scale }\end{array}$ & Web survey & PSUS \\
\hline $\begin{array}{c}\text { Asem A. } \\
\text { Alageel [47], } \\
\text { worldwide, } \\
2021\end{array}$ & 506 & $\begin{array}{l}\text { Postgraduate } \\
\text { students }\end{array}$ & $\begin{array}{c}\text { Age } 21 \text { years and } \\
\text { above } \\
\begin{array}{c}(21-24=9.41 \% \\
25-29=35.88 \% \\
30-39=44.51 \% \\
>=40=10.20 \%)\end{array}\end{array}$ & $\begin{array}{c}\text { Male }=158 \\
\text { Female }=348\end{array}$ & Cross-sectional & $\begin{array}{l}\text { Patient Health Questionnaire } \\
\text { (PHQ9) for depression, } \\
\text { Athens Insomnia Scale (AIS), } \\
\text { the Fagerström Test for } \\
\text { Cigarette Dependence } \\
\text { Questionnaire (FTCd),The } \\
\text { adult ADHD Self-Report } \\
\text { Scale (ASRS-v1.1) }\end{array}$ & Online & $\begin{array}{c}\text { Smartphone } \\
\text { Addiction Scale } \\
\text { (SAS) }\end{array}$ \\
\hline
\end{tabular}




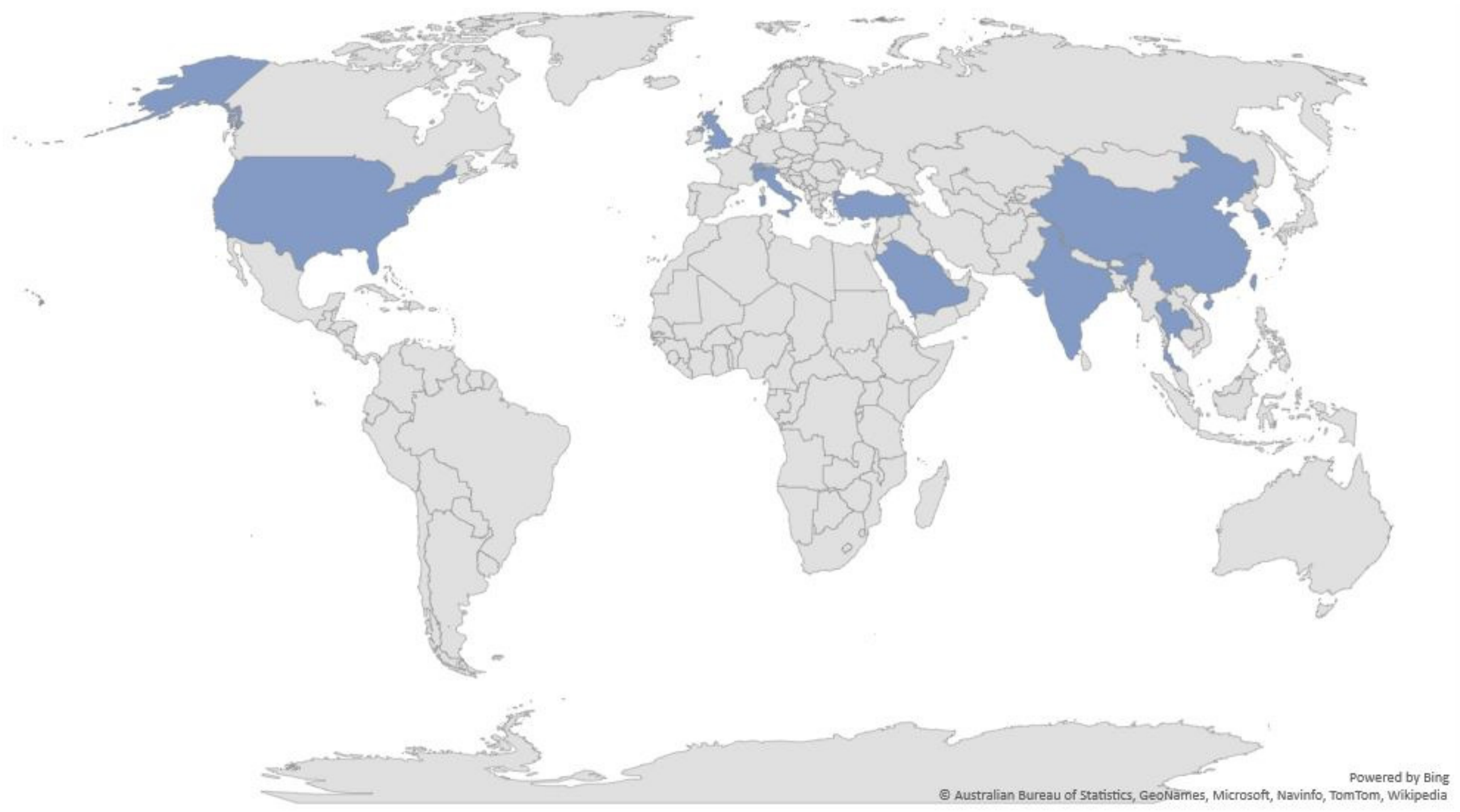

Figure 2. Global map indicating country of selected articles. 
Table 2. Summary of outcomes.

\begin{tabular}{|c|c|c|c|}
\hline Author and Reference & Outcomes & Specific Outcome & Quality \\
\hline HYE-JIN KIM [21] & $\begin{array}{l}\text { Smartphone addiction was significantly asso- } \\
\text { ciated with total accidents, falling/slipping, } \\
\text { and bumps/collisions }\end{array}$ & Accident & Fair \\
\hline Yeon-Jin Kim [22] & $\begin{array}{l}\text { - SA had a stronger relationship with depres- } \\
\text { sion and anxiety, stronger than IA }\end{array}$ & Depression and anxiety & Fair \\
\hline DEOKJONG LEE [23] & $\begin{array}{l}\text { Small GMV in the lateral orbitofrontal cortex } \\
\text { (OFC) was correlated with an increasing ten- } \\
\text { dency to be immersed in smartphone use }\end{array}$ & Gray matter abnormalities & Fair \\
\hline JeonHyeong Lee [24] & $\begin{array}{l}\text { - Significant differences in the cervical reposi- } \\
\text { tioning errors of flexion, extension, and right } \\
\text { and left lateral flexion were found among the } \\
\text { Normal Group, Moderate Addiction Group, } \\
\text { and Severe Addiction Group }\end{array}$ & Musculoskeletal problems & Fair \\
\hline Kyung Eun Lee [25] & $\begin{array}{l}\text { For both men and women, increases in smart- } \\
\text { phone dependency were associated with in- } \\
\text { creased anxiety scores }\end{array}$ & Anxiety & Fair \\
\hline Yeon-Seop Lee [26] & $\begin{array}{l}\text { Using smartphones continuously over long pe- } \\
\text { riods raises pressure on the median nerve and } \\
\text { increases the probability of occurrence of CTS }\end{array}$ & Carpal tunnel syndrome & Poor \\
\hline Mi Jung Rho [27] & $\begin{array}{l}\text { Mental health problems were related to } \\
\text { problematic smartphone use: }(1) \text { self-control }(66 \%) \text {, } \\
\text { (2) anxiety }(25 \%),(3) \text { depression }(7 \%) \text {, and }(4) \\
\text { dysfunctional impulsivities }(3 \%)\end{array}$ & Psychiatric symptoms & Fair \\
\hline & $\begin{array}{l}\text { - Significantly higher smartphone addiction } \\
\text { scores were associated with younger aged users. }\end{array}$ & & \\
\hline Aljohara A. Alhassan [28] & $\begin{array}{l}\text { Factors associated with higher depression scores } \\
\text { were high school-educated users }(\beta=-2.03 \text {, adj. } \\
p=0.01) \text { compared to the university educated } \\
\text { group and users with higher smart phone } \\
\text { addiction scores }(\beta=0.194 \text {, adj. } p<0.001) \text {. }\end{array}$ & Depression & Fair \\
\hline
\end{tabular}

- $\quad$ At least $43 \%$ had decreased sleeping hours Alosaimi, F. D. [29] and experienced a lack of energy the next day, $30 \%$ had an unhealthy lifestyle (ate more fast food, gained weight, and exercised less)

- A significant positive correlation was found between PUMP score and depression and trait anxiety scores, duration of owning a smartphone, and average duration of each daily call.

- $35.9 \%$ of our sample reported that they felt tired during day due to late-night smartphone use,

Jon D. Elhai [31]

- $\quad 38.1 \%$ of them acknowledged that their sleep quality decreased, and

Anxiety

Good

- $\quad 35.8 \%$ admitted that they slept less than four hours due to smartphone use more than once 
Table 2. Cont.

\begin{tabular}{|c|c|c|c|}
\hline Author and Reference & Outcomes & Specific Outcome & Quality \\
\hline Yuanming $\mathrm{Hu}$ [32] & $\begin{array}{l}\text { A primary understanding of white matter } \\
\text { characteristics in SPD indicated that the struc- } \\
\text { tural deficits might link to behavioral impair- } \\
\text { ments }\end{array}$ & Lower white matter integrity & Fair \\
\hline Jon D. Elhai [33] & $\begin{array}{l}\text { - COVID-19 anxiety correlated with severity of } \\
\text { PSU, depression, and anxiety } \\
12 \% \text { of participants were identified with at } \\
\text { least moderate depression, and } 24 \% \text { with } \\
\text { moderate anxiety }\end{array}$ & COVID-19 anxiety & Good \\
\hline Linbo Zhuang [34] & $\begin{array}{l}\text { - Cervical disc degeneration may be associated } \\
\text { with excessive smartphone use }\end{array}$ & cervical disc degeneration & Good \\
\hline Yasemin P. Demir [35] & $\begin{array}{l}\text { - There was a negative correlation between MP- } \\
\text { PUS and PSQI }(\mathrm{r}=-0.367, p \text { less than } 0.05) ; \mathrm{a} \\
\text { strong positive correlation between MPPUS } \\
\text { and ESS ( } \mathrm{r}=0.675, p \text { less than } 0.05) ; \text { and a neg- } \\
\text { ative correlation between MPPUS and } 24-\mathrm{h} \\
\text { MQoLQ }(\mathrm{r}=-0.508, p \text { less than } 0.05)\end{array}$ & $\begin{array}{l}\text { Increased headache duration, } \\
\text { poor sleep quality }\end{array}$ & Fair \\
\hline KADİR DEMİRCİ [36] & $\begin{array}{l}\text { - Smartphone Addiction Scale scores of females } \\
\text { were significantly higher than those of males } \\
\text { Depression, anxiety, and daytime dysfunction } \\
\text { scores were higher in the high smartphone } \\
\text { use group than in the low smartphone use } \\
\text { group }\end{array}$ & $\begin{array}{l}\text { Depression, anxiety, and } \\
\text { daytime dysfunction }\end{array}$ & Fair \\
\hline
\end{tabular}

- There is a mild, significant, positive correlation between the PU and LSAS scores of the students who participated in the study

- No significant relationship was found be-

Ayse Gokce [37] $\quad$ tween the PU and EAT scores in the study Increased smoking Fair

- Problematic Mobile Phone Use Scale total scores showed a significant correlation with smoking

- Frequency of poor sleep quality was significantly higher in students with smartphone addiction compared to others

S HariPriya [39]
- A moderately positive significant correlation between smartphone addiction and sleep quality was shown
Poor sleep quality, less physical activity

Good

- With addiction to smartphones, higher risk of psychological distress and poor sleep quality was found, which is inconsistent with a previous report that more and more young adults report poor sleep quality in a higher

Psychological distress, poor sleep quality

Good percentage when they become addicted to smartphones 
Table 2. Cont.

\begin{tabular}{|c|c|c|c|}
\hline Author and Reference & Outcomes & Specific Outcome & Quality \\
\hline Jon D. Elhai [42] & $\begin{array}{l}\text { Worry and anger may be helpful constructs in } \\
\text { understanding the phenomenology of PSU, } \\
\text { and psychological interventions for worry } \\
\text { and anger may offset PSU }\end{array}$ & Worry and anger & Good \\
\hline Matteo Megna [43] & $\begin{array}{l}\text { - Smartphone overuse was found to be linked } \\
\text { with higher signs of inflammation }\end{array}$ & Psoriatic arthritis & Fair \\
\hline $\begin{array}{c}\text { Arunrat } \\
\text { TangmunkongvorakulI [44] }\end{array}$ & $\begin{array}{l}\text { Female students had scores for psycholog- } \\
\text { ical well-being that were, on average, } 1.24 \\
\text { points higher than the scores of male students } \\
(p<0.001)\end{array}$ & Psychological well-being & Fair \\
\hline Zaheer Hussain [45] & $\begin{array}{l}\text { - The average time spent on a smartphone per } \\
\text { day was } 190.6 \mathrm{~min}(\mathrm{SD}=138.6) \\
\text { Problematic smartphone use was positively } \\
\text { related to time spent on the smartphone } \\
\text { and anxiety }\end{array}$ & Anxiety & Good \\
\hline MILES RICHARDSON [46] & $\begin{array}{l}\text { - PSUS was not found to have diagnostic ability } \\
\text { for high levels of anxiety }\end{array}$ & $\begin{array}{l}\text { Connectedness with nature } \\
\text { and anxiety }\end{array}$ & Fair \\
\hline Asem A. Alageel [47] & $\begin{array}{l}\text { - } 65.9 \% \text { of the participants who were identified } \\
\text { as having high smartphone use had no depres- } \\
\text { sion, whereas } 10.3 \% \text { had severe depression, } \\
16.1 \% \text { had moderately severe depression, and } \\
7.7 \% \text { had moderate depression } \\
\text { A significant correlation between the severity } \\
\text { of insomnia and smartphone use } \\
47.8 \% \text { of the participants with high smart- } \\
\text { phone use had ADHD symptoms }\end{array}$ & $\begin{array}{l}\text { Insomnia, depression, adult } \\
\text { ADHD }\end{array}$ & Fair \\
\hline
\end{tabular}

\subsection{Main Findings}

\subsubsection{Mental Health}

As outlined in Table 2, mental health was associated with SA in fourteen studies $[22,25,27,28,30,31,33,36,40-42,45-47]$. Depression and anxiety were the most common mental health conditions associated with SA [22,25,28,30,31,33,36,41,45,47]. Several depression measures were used; however, the Beck Depression Inventory was the most common measure used [28,30,36,40]. Alhassan et al. (2018) revealed that less-educated people and young adult users of the smartphone were at high risk of depression. Another study [28] found that the groups who were classified as smartphone-addicted had an increased risk of depression (relative risk 1.337; $p<0.001$ ) and anxiety (relative risk 1.402; $p<0.001$ ) [28]. Miles Richardson et al. (2018) found that problematic smartphone use (PSU) was positively related to anxiety [46].

Social anxiety was also associated with SA [41]. For instance, a study conducted in China during COVID-19 reported that COVID-19 anxiety was associated with the severity of problematic smartphone use [33].

Interestingly, female participants were more susceptible to SA [36] and showed significantly higher dependence on smartphones than men [25]. Further, a study conducted among university students in Thailand demonstrated that not only were female students more likely to be smartphone addicted, but smartphone addiction among female participants was likely to be negatively associated with psychological well-being [44]. 


\subsubsection{Physical Health \\ Musculoskeletal Problems}

The effect of SA on the musculoskeletal system was identified in four studies $[24,26,34,43]$ (Table 2). Among those studies, two studies reported cervical problems [24,34], one study demonstrated nerve thickness [26], and one study showed psoriatic arthritis [43]. Lee et al. (2014) compared cervical spine repositioning errors in different smartphone addiction groups and revealed that there were significant differences between non-addicted, moderately addicted, and severely addicted groups; the severe smartphone addict group showed the largest changes in posture, the cervical repositioning errors of flexion ( $3.2 \pm 0.8)$, extension $(4.9 \pm 1.1)$, right lateral flexion ( $3.9 \pm 1.0)$, and left lateral flexion ( $4.1 \pm 0.7)$. [24]. A study conducted among 2438 young patients suffering from chronic neck pain found that cervical disc degeneration was more likely to be associated with SA [34]. Another study conducted among university students revealed that excess smartphone use can cause nerve injury [26]. Megna et al. (2018) found that SA was linked to higher signs of inflammation in the musculoskeletal structures of hand joints.

\section{Sleep Quality and Sedentary Lifestyle}

Five studies showed an association between smartphone addiction and sleep quality $[29,35,38-40]$. The Pittsburgh Sleep Quality Index (PSQI) was used in all five studies (Table 1). A study conducted by Fahad et al. (2016) among 2367 university students reported $43 \%$ of the participants had decreased their sleeping hours due to SA, and $30 \%$ of the participants had an unhealthy lifestyle including weight gain, reduced exercise, and the consumption of more fast food when diagnosed with SA [29]. Another study conducted among migraine patients reported that SA can increase headache duration and decrease sleep quality [35].

\section{Accidents}

One study conducted by Hye-Jin Kim et al. (2017) revealed that SA is associated with different types of accidents, such as traffic accidents; falls/slips; bumps/collisions; being trapped in the subway, impalement, cuts, and exit wounds; and burns or electric shocks [21]. The study found that self-reported experience of accidents was significantly associated with SA [21].

\section{Neurological Problems}

Two studies reported the neurological effect of SA [23,32]; one study found alterations in white matter integrity [32] and another study reported smaller grey matter volume [23]. Hu et al. (2017) used a high-resolution magnetic resonance imaging technique to identify white matter integrity in young adults with SA and found that smartphone-addicted participants had significantly lower white matter integrity [32]. Lee et al. (2019) found that smartphone-addicted participants had significantly smaller grey matter volume (GMV) in the right lateral orbitofrontal cortex (OFC) [23].

\section{Discussion}

In recent years, several articles have examined the role of smartphone addiction and associated health outcomes among the adult population, however, substantial gaps still remain. To the best of our knowledge, no previous systematic review has been conducted to summarize these findings among this cohort. Our review is the first systematic review that utilizes empirical evidence from the last decades that demonstrates the relationship between smartphone addiction and health outcomes among adults. Interestingly, studies conducted in different parts of the world showed similar effects on health outcomes as a result of smartphone addiction. Hence, the consistency across the studies strengthens the study findings, emphasizing the association between SA and health outcomes.

Our findings suggest that depression and anxiety are significantly linked with smartphone addiction. One national USA survey found that $46 \%$ of smartphone owners believed 
they could not live without their phones [48]. Overuse patterns of smartphones involves a tendency to check notifications all the time, and such behavior patterns can induce "reassurance seeking" which broadly includes symptoms such as depression and anxiety [49]. This "reassurance seeking" pathway corresponds to those individuals whose smartphone use is driven by the necessity to maintain relationships and obtain reassurance from others. Bilieux and colleagues explained this reassurance-seeking behavior with the theoretical model of "problematic mobile phone use" [50]. In addition, this checking behavior is related to the next pathway, the "fear of missing out" (FOMO). One study found that FOMO mediated relations between both depression and anxiety severity with SA [51].

From our results, it is evident that musculoskeletal pain and insomnia are the two most common physical problems related to SA. Fingers, cervical, back, and shoulder problems are most commonly linked to excessive smartphone usage. Prolonged use of smartphones can cause defective postures such as forwarding head posture, which can produce injuries to the cervical spine and cause cervical pain [52]. Numerous studies found De Quervain tenosynovitis (characterized by pain in the wrist over the radio styloid process-the thumb side of wrist) was associated with different electronic devices like gaming controllers, tablets, and smartphones [53,54]. Texting and chatting through smartphones have been considered a risk factor for De Quervain tenosynovitis [55].

Poor sleep quality and difficulty in falling asleep or maintaining sleep has been identified as one of the negative consequences of SA, which is similar to our results $[56,57]$. Moreover, in line with our finding, another systematic review revealed that SA is related to poorer sleep quality [58]. One study found that $75 \%$ of the young adults (age $<30$ years) take their phones to bed, which may increase the likelihood of poor sleep quality [59]. Smartphone addicts are unsuccessful at controlling their smartphone use, even in bed. Again, fear of missing out could be the reason of taking phones in the beds as they do not want to miss any notification [60,61]. In addition, blue light emitted by smartphones can have a negative effect on circadian rhythms, leading to negative sleep consequences, such as going to sleep later than intended and thus reducing overall sleep time [62].

The neurological effect of SA is not clear yet from this review. However, currently neuroimaging studies play an important role in understanding the complexity of addictive behavior [63], as they can assess any pathological change in the brain. Two studies in this review reported the negative changes in grey matter and white matter integrity in the brain with the assistance of neuroimaging (Table 2), which is similar to the neuropathy caused by substance abuse $[64,65]$ and Internet addiction $[66,67]$. However, the modest sample size and the lack of a clinical evaluation are the potential limitations of these studies [23,32].

This review indicates that smartphone addiction shares similar features with substance abuse. A consistent relationship has been demonstrated between SA and physical and mental health symptoms, including depression, anxiety, musculoskeletal problems, and poor sleep. However, smartphones have become a part of daily life, facilitating work, education, or entertainment. Therefore, it is important not only to utilize the advantages of the smartphone but also to reduce the negative consequences. To address SA in a proper way, a validated definition and consistent diagnostic criteria of SA is required. The findings from this research suggest that healthcare providers and policymakers should recognize the problem and take necessary steps in raising community awareness about SA and its physical and mental impact.

\section{Limitations}

This systematic review has several limitations. First, all of the selected studies were cross-sectional (Table 1), therefore drawing conclusions about causal directions of associations is not possible. Secondly, all the papers were excluded if not in the English language; however, SA has received attention in Asian and European countries, and findings may have been published in other languages. This may lead to exclusion of studies conducted in diverse cultures and may bias the results of the review. Thirdly, most of the studies that were qualified to be included in this review were performed in developed countries, which 
may question the generalizability our findings to developing countries. Finally, most of the outcomes were reported over less than one year of follow-up. No standard scale and cut-off scores were used for the determination of smartphone addiction.

\section{Conclusions}

The current review describes the effect of smartphones on health outcomes in the adult population. Although the diagnostic criteria and effect of smartphone addiction are yet to be fully established, this review provides invaluable findings about the health impact of smartphone addiction and has significant implications for policy and decision makers. There is a need for more longitudinal studies to validate and strengthen this review's findings.

Supplementary Materials: The following are available online at https:/ /www.mdpi.com/article/10 .3390/ijerph182212257/s1, Table S1. Electronic search strategy.

Author Contributions: Z.A.R. conceptualized and designed the study, conducted initial searches, assessed the eligibility of the retrieved papers in the titles/abstracts and full text. S.B.Z. and M.S.A. independently reviewed all the retrieved abstracts and selected eligible papers. Z.A.R., A.-M.P., S.B.Z., M.S.A. and H.H. critically assessed the eligible studies and extracted data. Z.A.R. analyzed and interpreted the data and drafted the manuscript. All authors critically reviewed the manuscript. A.-M.P. and H.H. reviewed and approved the final manuscript. All authors have read and agreed to the published version of the manuscript.

Funding: This research received no funding.

Institutional Review Board Statement: Not applicable.

Informed Consent Statement: Not applicable.

Data Availability Statement: Not applicable.

Conflicts of Interest: Authors declared no conflict of interest.

\section{References}

1. Wajcman, J. Life in the fast lane? Towards a sociology of technology and time. Br. J. Sociol. 2008, 59, 59-77. [CrossRef] [PubMed]

2. Sharma, A.; Grant, D. Narrative, drama and charismatic leadership: The case of Apple's Steve Jobs. Leadership 2011, 7, 3-26. [CrossRef]

3. Ozdalga, E.; Ozdalga, A.; Ahuja, N. The Smartphone in Medicine: A Review of Current and Potential Use Among Physicians and Students. J. Med. Internet Res. 2012, 14, e128. [CrossRef]

4. Zargaran, A.; Ash, J.; Kerry, G.; Rasasingam, D.; Gokani, S.; Mittal, A.; Zargaran, D. Ethics of Smartphone Usage for Medical Image Sharing. Indian J. Surg. 2018, 80, 300-301. [CrossRef]

5. Karim, R.; Chaudhri, P. Behavioral Addictions: An Overview. J. Psychoact. Drugs 2012, 44, 5-17. [CrossRef] [PubMed]

6. Grant, J.E.; Potenza, M.N.; Weinstein, A.; Gorelick, D.A. Introduction to behavioral addictions. Am. J. Drug Alcohol Abus. 2010, 36, 233-241. [CrossRef]

7. Young, K.S. Internet Addiction: A New Clinical Phenomenon and Its Consequences. Am. Behav. Sci. 2004, 48, 402-415. [CrossRef]

8. Kwon, M.; Lee, J.-Y.; Won, W.-Y.; Park, J.-W.; Min, J.-A.; Hahn, C.; Gu, X.; Choi, J.-H.; Kim, D.-J. Development and Validation of a Smartphone Addiction Scale (SAS). PLoS ONE 2013, 8, e56936. [CrossRef]

9. Montag, C.; Wegmann, E.; Sariyska, R.; Demetrovics, Z.; Brand, M. How to overcome taxonomical problems in the study of Internet use disorders and what to do with "smartphone addiction"? J. Behav. Addict. 2021, 9, 908-914. [CrossRef]

10. Watkins, S.C. The Young and The Digital: What the Migration to Social-Network Sites, Games, and Anytime, Anywhere Media Means for Our Future; Beacon Press: Boston, MA, USA, 2009.

11. Vuori, T.O.; Huy, Q.N. Distributed Attention and Shared Emotions in the Innovation Process: How Nokia Lost the Smartphone Battle. Adm. Sci. Q. 2015, 61, 9-51. [CrossRef]

12. Ellis, D.A. Are smartphones really that bad? Improving the psychological measurement of technology-related behaviors. Comput. Hum. Behav. 2019, 97, 60-66. [CrossRef]

13. Alavi, S.S.; Ferdosi, M.; Jannatifard, F.; Eslami, M.; Alaghemandan, H.; Setare, M. Behavioral Addiction versus Substance Addiction: Correspondence of Psychiatric and Psychological Views. Int. J. Prev. Med. 2012, 3, 290-294. [PubMed]

14. Van Rooij, A.J.; Prause, N. A critical review of "Internet addiction" criteria with suggestions for the future. J. Behav. Addict. 2014, 3, 203-213. [CrossRef] [PubMed]

15. Petry, N.M.; Zajac, K.; Ginley, M.K. Behavioral Addictions as Mental Disorders: To Be or Not to Be? Annu. Rev. Clin. Psychol. 2018, 14, 399-423. [CrossRef] 
16. Al-Hadidi, F.; Bsisu, I.; Al Ryalat, S.A.; Al-Zu'Bi, B.; Bsisu, R.; Hamdan, M.; Kanaan, T.; Yasin, M.; Samarah, O. Association between mobile phone use and neck pain in university students: A cross-sectional study using numeric rating scale for evaluation of neck pain. PLoS ONE 2019, 14, e0217231. [CrossRef]

17. Horvath, J.; Mundinger, C.; Schmitgen, M.M.; Wolf, N.D.; Sambataro, F.; Hirjak, D.; Kubera, K.M.; Koenig, J.; Wolf, R.C. Structural and functional correlates of smartphone addiction. Addict. Behav. 2020, 105, 106334. [CrossRef]

18. Lin, Y.-H.; Chang, L.-R.; Lee, Y.-H.; Tseng, H.-W.; Kuo, T.B.J.; Chen, S.-H. Development and Validation of the Smartphone Addiction Inventory (SPAI). PLoS ONE 2014, 9, e98312. [CrossRef]

19. Panova, T.; Carbonell, X. Is smartphone addiction really an addiction? J. Behav. Addict. 2018, 7, 252-259. [CrossRef]

20. NIH. Study Quality Assessment Tools. 2021. Available online: https://www.nhlbi.nih.gov/health-topics/study-qualityassessment-tools (accessed on 2 March 2021).

21. Kim, H.J.; Min, J.-Y.; Kim, H.-J.; Min, K.-B. Accident risk associated with smartphone addiction: A study on university students in Korea. J. Behav. Addict. 2017, 6, 699-707. [CrossRef]

22. Kim, Y.-J.; Jang, H.M.; Lee, Y.; Lee, D.; Kim, D.-J. Effects of Internet and Smartphone Addictions on Depression and Anxiety Based on Propensity Score Matching Analysis. Int. J. Environ. Res. Public Health 2018, 15, 859. [CrossRef]

23. Lee, D.; Namkoong, K.; Lee, J.; Lee, B.O.; Jung, Y.-C. Lateral orbitofrontal gray matter abnormalities in subjects with problematic smartphone use. J. Behav. Addict. 2019, 8, 404-411. [CrossRef]

24. Lee, J.; Seo, K. The Comparison of Cervical Repositioning Errors According to Smartphone Addiction Grades. J. Phys. Ther. Sci. 2014, 26, 595-598. [CrossRef] [PubMed]

25. Lee, K.E.; Kim, S.-H.; Ha, T.-Y.; Yoo, Y.-M.; Han, J.-J.; Jung, J.-H.; Jang, J.-Y. Dependency on Smartphone Use and its Association with Anxiety in Korea. Public Health Rep. 2016, 131, 411-419. [CrossRef] [PubMed]

26. Lee, Y.-S.; Yang, H.-S.; Jeong, C.-J.; Yoo, Y.-D.; Jeong, G.-Y.; Moon, J.-S.; Kang, M.-K.; Hong, S.-W. Changes in the Thickness of Median Nerves Due to Excessive Use of Smartphones. J. Phys. Ther. Sci. 2012, 24, 1259-1262. [CrossRef]

27. Rho, M.J.; Park, J.; Na, E.; Jeong, J.-E.; Kim, J.K.; Kim, D.-J.; Choi, I.Y. Types of problematic smartphone use based on psychiatric symptoms. Psychiatry Res. 2019, 275, 46-52. [CrossRef] [PubMed]

28. Alhassan, A.A.; Alqadhib, E.M.; Taha, N.W.; Alahmari, R.A.; Salam, M.; Almutairi, A.F. The relationship between addiction to smartphone usage and depression among adults: A cross sectional study. BMC Psychiatry 2018, 18, 1-8. [CrossRef] [PubMed]

29. Alosaimi, F.D.; Alyahya, H.; Alshahwan, H.; Al Mahyijari, N.; Shaik, S.A. Smartphone addiction among university students in Riyadh, Saudi Arabia. Saudi Med. J. 2016, 37, 675-683. [CrossRef]

30. El-Sayed Desouky, D.; Abu-Zaid, H. Mobile phone use pattern and addiction in relation to depression and anxiety. East Mediterr. Health J. 2020, 26, 692-699. [CrossRef]

31. Elhai, J.D.; Yang, H.; Fang, J.; Bai, X.; Hall, B. Depression and anxiety symptoms are related to problematic smartphone use severity in Chinese young adults: Fear of missing out as a mediator. Addict. Behav. 2019, 101, 105962. [CrossRef]

32. Hu, Y.; Long, X.; Lyu, H.; Zhou, Y.; Chen, J. Alterations in White Matter Integrity in Young Adults with Smartphone Dependence. Front. Hum. Neurosci. 2017, 11, 532. [CrossRef]

33. Elhai, J.D.; Yang, H.; McKay, D.; Asmundson, G.J. COVID-19 anxiety symptoms associated with problematic smartphone use severity in Chinese adults. J. Affect. Disord. 2020, 274, 576-582. [CrossRef]

34. Zhuang, L.; Wang, L.; Xu, D.; Wang, Z.; Liang, R. Association between excessive smartphone use and cervical disc degeneration in young patients suffering from chronic neck pain. J. Orthop. Sci. 2021, 26, 110-115. [CrossRef] [PubMed]

35. Demir, Y.P.; Sümer, M.M. Effects of smartphone overuse on headache, sleep and quality of life in migraine patients. Neurosciences 2019, 24, 115-121. [CrossRef]

36. Demirci, K.; Akgönül, M.; Akpinar, A. Relationship of smartphone use severity with sleep quality, depression, and anxiety in university students. J. Behav. Addict. 2015, 4, 85-92. [CrossRef] [PubMed]

37. Gokce, A.; Ozer, A. The relationship between problematic cell phone use, eating disorders and social anxiety among university students. Pak. J. Med. Sci. 2021, 37, 1201-1205. [CrossRef] [PubMed]

38. Ozcan, B.; Acimis, N.M. Sleep Quality in Pamukkale University Students and its relationship with smartphone addiction. Pak. J. Med. Sci. 2020, 37, 206-211. [CrossRef]

39. Haripriya, S.; Samuel, S.E.; Megha, M. Correlation between Smartphone Addiction, Sleep Quality and Physical Activity among Young Adults. J. Clin. Diagn. Res. 2019, 13, YC05-YC09. [CrossRef]

40. Lane, H.-Y.; Chang, C.-J.; Huang, C.-L.; Chang, Y.-H. An Investigation into Smartphone Addiction with Personality and Sleep Quality among University Students. Int. J. Environ. Res. Public Health 2021, 18, 7588. [CrossRef]

41. Annoni, A.; Petrocchi, S.; Camerini, A.-L.; Marciano, L. The Relationship between Social Anxiety, Smartphone Use, Dispositional Trust, and Problematic Smartphone Use: A Moderated Mediation Model. Int. J. Environ. Res. Public Health 2021, $18,2452$. [CrossRef]

42. Elhai, J.D.; Rozgonjuk, D.; Yildirim, C.; Alghraibeh, A.M.; Alafnan, A.A. Worry and anger are associated with latent classes of problematic smartphone use severity among college students. J. Affect. Disord. 2019, 246, 209-216. [CrossRef]

43. Megna, M.; Gisonni, P.; Napolitano, M.; Orabona, G.D.; Patruno, C.; Ayala, F.; Balato, N. The effect of smartphone addiction on hand joints in psoriatic patients: An ultrasound-based study. J. Eur. Acad. Dermatol. Venereol. 2018, 32, 73-78. [CrossRef] 
44. Tangmunkongvorakul, A.; Musumari, P.M.; Thongpibul, K.; Srithanaviboonchai, K.; Techasrivichien, T.; Suguimoto, S.P.; OnoKihara, M.; Kihara, M. Association of excessive smartphone use with psychological well-being among university students in Chiang Mai, Thailand. PLoS ONE 2019, 14, e0210294. [CrossRef]

45. Hussain, Z.; Griffiths, M.D.; Sheffield, D. An investigation into problematic smartphone use: The role of narcissism, anxiety, and personality factors. J. Behav. Addict. 2017, 6, 378-386. [CrossRef] [PubMed]

46. Richardson, M.; Hussain, Z.; Griffiths, M.D. Problematic smartphone use, nature connectedness, and anxiety. J. Behav. Addict. 2018, 7, 109-116. [CrossRef]

47. Alageel, A.A.; Alyahya, R.A.; Bahatheq, Y.A.; Alzunaydi, N.A.; Alghamdi, R.A.; Alrahili, N.M.; McIntyre, R.S.; Iacobucci, M. Smartphone addiction and associated factors among postgraduate students in an Arabic sample: A cross-sectional study. BMC Psychiatry 2021, 21, 1-10. [CrossRef] [PubMed]

48. Smith, A.; McGeeney, K.; Duggan, M. US Smartphone Use in 2015; Report; Pew Research Center: Washington, DC, USA, 2015.

49. Ratan, Z.A.; Bin Zaman, S.; Islam, S.M.S.; Hosseinzadeh, H. Smartphone overuse: A hidden crisis in COVID-19. Health Policy Technol. 2021, 10, 21-22. [CrossRef] [PubMed]

50. Billieux, J.; Maurage, P.; Lopez-Fernandez, O.; Kuss, D.J.; Griffiths, M.D. Can Disordered Mobile Phone Use Be Considered a Behavioral Addiction? An Update on Current Evidence and a Comprehensive Model for Future Research. Curr. Addict. Rep. 2015, 2, 156-162. [CrossRef]

51. Wolniewicz, C.A.; Rozgonjuk, D.; Elhai, J.D. Boredom proneness and fear of missing out mediate relations between depression and anxiety with problematic smartphone use. Hum. Behav. Emerg. Technol. 2020, 2, 61-70. [CrossRef]

52. Elserty, N.S.; Helmy, N.A.; Mounir, K.M. Smartphone addiction and its relation to musculoskeletal pain in Egyptian physical therapy students. Eur. J. Physiother. 2020, 22, 70-78. [CrossRef]

53. Baabdullah, A.; Bokhary, D.; Kabli, Y.; Saggaf, O.; Daiwali, M.; Hamdi, A. The association between smartphone addiction and thumb/wrist pain: A cross-sectional study. Medicine 2020, 99, e19124. [CrossRef]

54. Saito, K.; Saito, Y. Relationship between Information and Communication Device Usage and Development of Hand Disorders. Inq. J. Health Care Organ. Provis. Financ. 2021, 58, 00469580211029607. [CrossRef]

55. Benites-Zapata, V.A.; Jiménez-Torres, V.E.; Ayala-Roldán, M.P. Problematic smartphone use is associated with de Quervain's tenosynovitis symptomatology among young adults. Musculoskelet. Sci. Pract. 2021, 53, 102356. [CrossRef]

56. Elhai, J.D.; Levine, J.C.; Dvorak, R.D.; Hall, B. Fear of missing out, need for touch, anxiety and depression are related to problematic smartphone use. Comput. Hum. Behav. 2016, 63, 509-516. [CrossRef]

57. Zhang, M.X.; Wu, A.M.S. Effects of smartphone addiction on sleep quality among Chinese university students: The mediating role of self-regulation and bedtime procrastination. Addict. Behav. 2020, 111, 106552. [CrossRef] [PubMed]

58. Sohn, S.Y.; Rees, P.; Wildridge, B.; Kalk, N.J.; Carter, B. Prevalence of problematic smartphone usage and associated mental health outcomes amongst children and young people: A systematic review, meta-analysis and GRADE of the evidence. BMC Psychiatry $2019,19,356$.

59. Alshobaili, F.A.; Alyousefi, N.A. The effect of smartphone usage at bedtime on sleep quality among Saudi non- medical staff at King Saud University Medical City. J. Fam. Med. Prim. Care 2019, 8, 1953-1957. [CrossRef]

60. Li, L.; Griffiths, M.D.; Mei, S.; Niu, Z. Fear of Missing Out and Smartphone Addiction Mediates the Relationship Between Positive and Negative Affect and Sleep Quality Among Chinese University Students. Front. Psychiatry 2020, 11, 877. [CrossRef]

61. Zhang, M.X.; Zhou, H.; Yang, H.M.; Wu, A.M.S. The prospective effect of problematic smartphone use and fear of missing out on sleep among Chinese adolescents. Curr. Psychol. 2021, 1-9. [CrossRef]

62. Höhn, C.; Schmid, S.; Plamberger, C.; Bothe, K.; Angerer, M.; Gruber, G.; Pletzer, B.; Hoedlmoser, K. Preliminary Results: The Impact of Smartphone Use and Short-Wavelength Light during the Evening on Circadian Rhythm, Sleep and Alertness. Clocks Sleep 2021, 3, 66-86. [CrossRef]

63. Bahji, A.; Brietzke, E.; Soares, C.; Stuart, H. Recent Advances in Biomarkers of Addiction: A Narrative Review. Can. J. Addict. 2021, 12, 6-12. [CrossRef]

64. Hanlon, C.; Canterberry, M. The use of brain imaging to elucidate neural circuit changes in cocaine addiction. Subst. Abus. Rehabil. 2012, 3, 115-128. [CrossRef] [PubMed]

65. Yeh, P.-H.; Simpson, K.; Durazzo, T.C.; Gazdzinski, S.; Meyerhoff, D. Tract-based spatial statistics (TBSS) of diffusion tensor imaging data in alcohol dependence: Abnormalities of the motivational neurocircuitry. Psychiatry Res. Neuroimag. 2009, 173, 22-30. [CrossRef] [PubMed]

66. Yuan, K.; Qin, W.; Wang, G.; Zeng, F.; Zhao, L.; Yang, X.; Liu, P.; Liu, J.; Sun, J.; Von Deneen, K.M.; et al. Microstructure Abnormalities in Adolescents with Internet Addiction Disorder. PLoS ONE 2011, 6, e20708. [CrossRef]

67. Zhou, Y.; Lin, F.-C.; Du, Y.-S.; Qin, L.-D.; Zhao, Z.-M.; Xu, J.-R.; Lei, H. Gray matter abnormalities in Internet addiction: A voxel-based morphometry study. Eur. J. Radiol. 2011, 79, 92-95. [CrossRef] [PubMed] 